\title{
Existence of positive solutions for p-Laplacian systems involving left and right fractional derivatives
}

Positive
solutions for
p-Laplacian
systems

235

Samira Ramdane

École Normale Supérieure d'Enseignement Technologique de Skikda,

Skikda, Algeria, and

Assia Guezane-Lakoud

Laboratory of Advanced Material, Faculty of Sciences,

Badji Mokhtar-Annaba University, Annaba, Algeria

\begin{abstract}
Purpose - The paper deals with the existence of positive solutions for a coupled system of nonlinear fractional differential equations with p-Laplacian operator and involving both right Riemann-Liouville and left Caputotype fractional derivatives. The existence results are obtained by the help of Guo-Krasnosel'skii fixed-point theorem on a cone in the sublinear case. In addition, an example is included to illustrate the main results.

Design/methodology/approach - Fixed-point theorems.
\end{abstract}

Findings - No finding.

Originality/value - The obtained results are original.

Keywords Fractional derivatives, Integral condition, Existence of solutions, Fixed point theorem

Paper type Research paper

\section{Introduction}

In this paper, we consider the following coupled system of nonlinear fractional differential equations with p-Laplacian operator:

$$
\left\{\begin{array}{l}
{ }^{R} D_{1^{-}}^{\alpha} \phi_{p}\left({ }^{C} D_{0^{+}}^{\beta_{1}} u(t)\right)+a_{1}(t) f_{1}(u(t), v(t))=0, \quad t \in[0,1], \\
{ }^{R} D_{1^{-}}^{\alpha} \phi_{p}\left({ }^{C} D_{0^{+}}^{\beta_{2}} v(t)\right)+a_{2}(t) f_{2}(u(t), v(t))=0, \quad t \in[0,1], \\
\phi_{p}\left({ }^{C} D_{0^{+}}^{\beta_{1}} u(1)\right)=0, u^{\prime}(0)=0, \eta_{1} u(1)-u(0)=\int_{0}^{1} g_{1}(s, u(s), v(s)) d s, \\
\phi_{p}\left({ }^{C} D_{0^{+}}^{\beta_{2}} v(1)\right)=0, v^{\prime}(0)=0, \eta_{2} v(1)-v(0)=\int_{0}^{1} g_{2}(s, u(s), v(s)) d s .
\end{array}\right.
$$

\section{JEL Classification - 34A08, 34B15}

(C) Samira Ramdane and Assia Guezane-Lakoud. Published in Arab Journal of Mathematical Sciences. Published by Emerald Publishing Limited. This article is published under the Creative Commons Attribution (CCBY 4.0) licence. Anyone may reproduce, distribute, translate and create derivative works of this article (for both commercial and non-commercial purposes), subject to full attribution to the original publication and authors. The full terms of this licence may be seen at http://creativecommons. org/licences/by/4.0/legalcode

The authors are grateful to the anonymous referees for their valuable comments and suggestions, which helped to improve the quality of the paper.

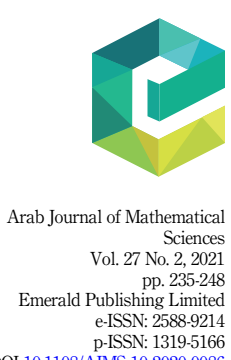


AJMS

27,2

236

where $0<\alpha<1,1<\beta_{i}<2, \eta_{i}>1,(i=1,2)$ and $\phi_{p}(s)=|s|^{p-2} s, p>1, \phi_{q}=\left(\phi_{p}\right)^{-1}$, $\frac{1}{p}+\frac{1}{q}=1,{ }^{\mathbb{R}} D_{1^{-}}^{\alpha}$ the right Riemann-Liouville fractional derivative, ${ }^{C} D_{0^{+}}^{\beta_{i}}$ denotes the left Caputo fractional derivative of order $\beta_{i}$, the functions $a_{i} \in C\left([0,1], \mathbb{R}^{+}\right), f_{i} \in C\left(\mathbb{R}^{+} \times \mathbb{R}^{+}\right.$, $\left.\mathbb{R}^{+}\right), g_{i} \in C\left([0,1] \times \mathbb{R}^{+} \times \mathbb{R}^{+}, \mathbb{R}^{+}\right)$for $i=1,2$.

Fractional differential equations arise in many engineering and scientific disciplines as the mathematical modeling of systems and processes in the fields of physics, chemistry, aerodynamics, electrodynamics of a complex medium, polymer rheology, etc. Fractional differential equations also serve as an excellent tool for the description of hereditary properties of various materials and processes. For the basic theory and recent development of subject, see [1,2,3]. Recently, a linear boundary value problem involving both the right Caputo and the left Riemann-Liouville fractional derivatives have been studied by many authors $[4,5]$ Many people pay attention to the existence and multiplicity of solutions or positive solutions for boundary value problems of nonlinear fractional differential equations by means of some fixed-point theorems [6-13].

In [14], by applying Guo-Krasnosel'skiı's fixed-point theorem, Guezane-Lakoud and Ashyralyev discussed the existence of positive solutions for the following fractional BVP

$$
\left\{\begin{array}{l}
D_{0^{+}}^{q} u(t)=f(t, u(t)), t \in[0,1], 1<q<2 \\
u^{\prime}(0)=0, u(0)-\alpha u(1)=\int_{0}^{1} g(s, u(s)) d s .
\end{array}\right.
$$

where $f:[0,1] \times \mathbb{R} \rightarrow \mathbb{R}$ is a given function, $\alpha \in \mathbb{R}^{+}, D_{0^{+}}^{q}$ denotes the Caputo's fractional derivative of order $q$.

On the other hand, the study of coupled systems involving fractional differential equations is also important as such systems occur in various problems, see $[13,15,16]$ and the references therein.

In the interesting paper [17], Liu studied by the help of Picard iterative method and Schaefer's fixed-point theorem, the existence of solutions for four classes of boundary value problems for impulsive fractional differential equations.

In [12], relying on the Guo-Krasnosel'skiîs fixed-point theorem, Li and Wei discussed existence of positive solutions for the following coupled system of mixed higher-order nonlinear singular fractional differential equations with integral boundary conditions

$$
\left\{\begin{array}{l}
D_{0^{+}}^{\alpha_{1}} u(t)+a_{1}(t) f_{1}(t, u(t), v(t))=0, t \in[0,1] \\
D_{0^{+}}^{\alpha_{2}} v(t)+a_{2}(t) f_{2}(t, u(t))=0, t \in[0,1] \\
u^{(j)}(0)=v^{(k)}(0)=0,0 \leq j \leq n_{1}-2,0 \leq k \leq n_{2}-2 \\
u(1)=\int_{0}^{1} h_{1}(s) u(s) d s, v(1)=\int_{0}^{1} h_{2}(s) v(s) d s
\end{array}\right.
$$

where $n_{i}-1<\alpha_{i}<n_{i}, n_{i} \geq 3, D_{0^{+}}^{\alpha_{i}}$ are the standard Riemann-Liouville fractional derivative, $a_{i}(t) \in C[0,1]$ may be singular at $t=0$, and/or $t=1, h_{i} \in L_{1}[0,1]$ are nonnegative $(i=1,2)$.

On the other hand, differential equations with $\mathrm{p}$-Laplacian operator have been widely studied owing to its importance in theory and application of mathematics and physics, such in non-Newtonian mechanics, nonlinear elasticity and glaciology, population biology, 
nonlinear flow laws. There are a very large number of papers devoted to the existence of solutions of the p-Laplacian operator, see for example [18-25].

In [26] G. Q. Chai, studied the existence of positive solutions for the boundary-value problem of nonlinear fractional differential equations with p-Laplacian operator

$$
\left\{\begin{array}{l}
D_{0^{+}}^{\beta} \phi_{p}\left(D_{0^{+}}^{\alpha} u(t)\right)+f(t, u(t))=0,0<t<1, \\
D_{0^{+}}^{\alpha} u(0)=0, D_{0^{+}}^{\alpha} u(1)+\sigma D_{0^{+}}^{\gamma} u(1)=0, u(0)=0 .
\end{array}\right.
$$

Positive solutions for p-Laplacian systems

where $1<\alpha<2,0<\beta<1, \phi_{p}(s)=|s|^{p-2} s, p>1, \phi_{q}=\left(\phi_{p}\right)^{-1}, \frac{1}{p}+\frac{1}{q}=1, D_{0^{+}}^{\alpha}, D_{0^{+}}^{\beta}$ are the standard Riemann-Liouville fractional derivatives, $0<\gamma \leq 1$, The function $f:[0,1] \times \mathbb{R}^{+} \rightarrow \mathbb{R}^{+}$is continuous.

The rest of the paper is organized as follows. In Section 2, we present preliminaries and lemmas. Section 3, we investigate the existence of a solution for the corresponding fractional linear boundary value problem. Finally, Section 4 is devoted to the existence of positive solutions under some sufficient conditions on the nonlinear terms, then we give an example to illustrate our results.

\section{Preliminaries}

In this section, we recall the basic definitions and lemmas from fractional calculus theory, see $[2,3]$, for more details.

Let $\alpha>0,[a, b]$ be a finite interval of $\mathbb{R}$ and $g$ a real function on $(a, b)$. The left and right Riemann-Liouville fractional integral of the function $g$ are defined, respectively, by

$$
I_{a+}^{\alpha} f(t)=\frac{1}{\Gamma(\alpha)} \int_{a}^{t}(t-s)^{\alpha-1} g(s) d s, \quad I_{b-}^{\alpha} f(t)=\frac{1}{\Gamma(\alpha)} \int_{t}^{b}(s-t)^{\alpha-1} g(s) d s,
$$

provided that the right-hand side exists.

The right Riemann-Liouville fractional derivative and the left Caputo fractional derivative of order $\alpha>0$ of $g$ are, respectively

$$
{ }^{R} D_{b^{-}}^{\alpha} f(t)=\left(\frac{-d}{d t}\right)^{n} I_{b-}^{n-\alpha} g(t), \quad{ }^{C} D_{a^{+}}^{\alpha} f(t)=I_{a^{+}}^{n-\alpha} g^{(n)}(t),
$$

where $n<\alpha<n+1, n=[\alpha]+1$, provided that the right-hand side exists.

For the properties of Riemann-Liouville fractional derivative and Caputo fractional derivative, we obtain the following statement. Let $u \in L^{1}(0,1)$ then

$$
\begin{gathered}
I_{1^{-}}^{\alpha R} D_{1^{-}}^{\alpha} u(t)=u(t)+\sum_{i=1}^{n} a_{i}(1-t)^{\alpha-i} \\
I_{0^{+}}^{\alpha C} D_{0^{+}}^{\alpha} u(t)=u(t)+\sum_{k=0}^{n-1} b_{k} t^{k}
\end{gathered}
$$

where $a_{i}, b_{k} \in \mathbb{R}, i=0, \ldots n$, and $k=0, \ldots n-1$.

We also need the following lemma and theorem to obtain our results.

Lemma 2.1. [26] Let $c>0, \gamma>0$. for any $x, y \in[0, c]$ we have

(1) if $\gamma>1$, then $\left|x^{\gamma}-y^{\gamma}\right| \leq \gamma c^{\gamma-1}|x-y|$,

(2) if $0<\gamma \leq 1$, then $\left|x^{\gamma}-y^{\gamma}\right| \leq|x-y|^{\gamma}$. 
AJMS

27,2

Theorem 2.1. [27] (Guo-Krasnoselskiı's) Let $E$ be a Banach space, and let $K \subset E$, be a cone. Assume $\Omega_{1}$ and $\Omega_{2}$ are open subsets of $E$ with $0 \in \Omega_{1}, \bar{\Omega}_{1} \subset \Omega_{2}$ and let $T: K \cap\left(\bar{\Omega}_{2} \backslash \Omega_{1}\right) \rightarrow K$, be a completely continuous operator such that

(1) $\|T u\| \leq\|u\|, u \in K \cap \partial \Omega_{1}$, and $\|T u\| \geq\|u\|, u \in K \cap \partial \Omega_{2}$, or

(2) $\|T u\| \geq\|u\|, u \in K \cap \partial \Omega_{1}$ and $\|T u\| \leq\|u\|, u \in K \cap \partial \Omega_{2}$.

Then $T$ has a fixed point in $K \cap\left(\bar{\Omega}_{2} \backslash \Omega_{1}\right)$

\section{Linear boundary value problem}

Lemma 3.1. Assume that $y \in C(0,1) \cap L_{1}(0,1)$ and $1<\beta_{i}<2, i=1,2$, the unique solution of the boundary value problem

$$
\begin{gathered}
{ }^{C} D_{0^{+}}^{\beta_{i}} u(t)+y(t)=0, \quad t \in[0,1], \\
u^{\prime}(0)=0, \eta_{i} u(1)-u(0)=\int_{0}^{1} g_{i}(s) d s
\end{gathered}
$$

is given by

$$
u(t)=\int_{0}^{1} G_{i}(t, s) y(s) d s+\frac{1}{\eta_{i}-1} \int_{0}^{1} g_{i}(s) d s
$$

where

$$
G_{i}(t, s)=\frac{1}{\Gamma\left(\beta_{i}\right)} \begin{cases}\frac{\eta_{i}}{\eta_{i}-1}(1-s)^{\beta_{i}-1}-(t-s)^{\beta_{i}-1}, & 0 \leq s \leq t \leq 1 . \\ \frac{\eta_{i}}{\eta_{i}-1}(1-s)^{\beta_{i}-1}, & 0 \leq t \leq s \leq 1 .\end{cases}
$$

Proof. We apply (2.2) to equation (3.1) to get

$$
u(t)=-I_{0^{+}}^{\beta_{i}} y(t)+c_{1}+c_{2} t, t \in[0,1]
$$

thanks to boundary condition (3.2) we obtain $c_{2}=0$, and

$$
c_{1}=\frac{1}{\eta_{i}-1}\left[\frac{\eta_{i}}{\Gamma\left(\beta_{i}\right)} \int_{0}^{1}(1-s)^{\beta_{i}-1} y(s) d s+\int_{0}^{1} g_{i}(s) d s\right] .
$$

So, the unique solution of the problem (3.1) is

$$
\begin{aligned}
u(t) & =\frac{1}{\Gamma\left(\beta_{i}\right)}\left[-\int_{0}^{t}(t-s)^{\beta_{i}-1} y(s) d s+\frac{\eta_{i}}{\eta_{i}-1} \int_{0}^{1}(1-s)^{\beta_{i}-1} y(s) d s\right]+\frac{1}{\eta_{i}-1} \int_{0}^{1} g_{i}(s) d s \\
& =\int_{0}^{1} G_{i}(t, s) y(s) d s+\frac{1}{\eta_{i}-1} \int_{0}^{1} g_{i}(s) d s .
\end{aligned}
$$

The proof is completed. 
Lemma 3.2. If $y \in C(0,1) \cap L_{1}(0,1)$, then the boundary value problem

$$
\begin{gathered}
{ }^{R} D_{1^{-}}^{\alpha} \phi_{p}\left({ }^{C} D_{0^{+}}^{\beta_{i}} u(t)\right)+y(t)=0,0 \leq t \leq 1 \\
\phi_{p}\left({ }^{C} D_{0^{+}}^{\beta_{i}} u(1)\right)=0 \\
u^{\prime}(0)=0, \quad \eta_{i} u(1)-u(0)=\int_{0}^{1} g_{i}(s) d s
\end{gathered}
$$

Positive solutions for p-Laplacian systems

239

has an unique solution

$$
u(t)=\int_{0}^{1} G_{i}(t, s) \phi_{q}\left(\int_{s}^{1} \frac{(\tau-s)^{\alpha-1}}{\Gamma(\alpha)} y(\tau) d \tau\right) d s+\frac{1}{\eta_{i}-1} \int_{0}^{1} g_{i}(s) d s
$$

where $G_{i}(t, s)$ is defined as (3.4).

Proof. From Eqs (3.6) and (2.1), we have

$$
\phi_{p}\left({ }^{C} D_{0^{+}}^{\beta_{i}} u(t)\right)=-I_{1^{-}}^{\alpha} y(t)+C_{1}(1-t)^{\alpha-1}, C_{1} \in \mathbb{R} .
$$

By the boundary conditions (3.7) we get $C_{1}=0$, consequently,

$$
\phi_{p}\left({ }^{C} D_{0^{+}}^{\beta_{i}} u(t)\right)=-I_{1^{-}}^{\alpha} y(t)
$$

and then

$$
{ }^{C} D_{0^{+}}^{\beta_{i}} u(t)+\phi_{q}\left(\frac{1}{\Gamma(\alpha)} \int_{t}^{1}(s-t)^{\alpha-1} y(s) d s\right)=0, t \in[0,1] .
$$

Thus, the fractional boundary value problem (3.1)-(3.2) is equivalent to the following problem

$$
\begin{aligned}
& { }^{C} D_{0^{+}}^{\beta_{i}} u(t)+\phi_{q}\left(\frac{1}{\Gamma(\alpha)} \int_{s}^{1}(s-t)^{\alpha-1} y(s) d s\right)=0, t \in[0,1] \\
& u^{\prime}(0)=0 ; \eta_{i} u(1)-u(0)=\int_{0}^{1} g_{i}(s) d s .
\end{aligned}
$$

Lemma 3.1 implies that the problem (3.6), (3.7) and (3.8) has an unique solution

$$
u(t)=\int_{0}^{1} G_{i}(t, s) \phi_{q}\left(\frac{1}{\Gamma(\alpha)} \int_{s}^{1}(\tau-s)^{\alpha-1} y(\tau) d \tau\right) d s+\frac{1}{\eta_{i}-1} \int_{0}^{1} g_{i}(s) d s,
$$

the proof is achieved.

Lemma 3.3. The functions $G_{i}(t, s), i=1,2$ are continuous on $[0,1] \times[0,1]$ and satisfy the following properties:

(1) $G_{i}(t, s)>0$ for $t, s \in[0,1), i=1,2$

(2) $\frac{1}{\eta_{i}} G_{i}(s, s) \leq G_{i}(t, s) \leq G_{i}(s, s), i=1,2$ for $(t, s) \in[0,1) \times[0,1)$. 
AJMS

27,2

\section{0}

Proof. (1) Observing the expression of $G_{i}(t, s)$, it is easy to see that $G_{i}(t, s)>0$, for $t, s \in[0,1), i=1,2$

(2) First, $G_{i}(t, s) \leq G_{i}(s, s)$ for $t, s \in[0,1)$

Second, setting

$$
\begin{aligned}
& g_{i, 1}(t, s)=\frac{\eta_{i}}{\left(\eta_{i}-1\right) \Gamma\left(\beta_{i}\right)}(1-s)^{\beta_{i}-1}-\frac{(t-s)^{\beta_{i}-1}}{\Gamma\left(\beta_{i}\right)}, s \leq t \\
& g_{i, 2}(s)=\frac{\eta_{i}}{\left(\eta_{i}-1\right) \Gamma\left(\beta_{i}\right)}(1-s)^{\beta_{i}-1}, t \leq s
\end{aligned}
$$

for given $s \in[0,1), g_{i, 1}(t, s)$ is decreasing as a function of $t$, then,

$$
\begin{aligned}
g_{i, 1}(t, s) & \geq g_{i, 1}(1, s) \\
& =\frac{1}{\left(\eta_{i}-1\right) \Gamma\left(\beta_{i}\right)}(1-s)^{\beta_{i}-1} \\
& \geq \frac{1}{\eta_{i}} G_{i}(s, s),
\end{aligned}
$$

and $g_{i, 2}(s) \geq \frac{1}{\eta_{i}} G_{i}(s, s)$.

\section{Existence of positive solutions}

We need to introduce some notations for the forthcoming discussion. Let $X=C[0,1] \times C[0,1]$ be the Banach space endowed with the norm

$$
\left\|\left(x_{1}, x_{2}\right)\right\|=\max \left(\left\|x_{i}\right\|_{\infty}, i=1,2\right)
$$

where $\left\|x_{i}\right\|_{\infty}=\max _{t \in[0,1]}\left|x_{i}(t)\right|$

Define the cone $P \subset X$ by

$$
P=\left\{\left(x_{1}, x_{2}\right) \in X: x_{i}(t) \geq 0, t \in[0,1], \min _{t \in[0,1]} x_{i}(t) \geq \frac{1}{\eta_{i}}\left\|x_{i}\right\|_{\infty}, i=1,2\right\}
$$

Let us introduce the following notations

$$
\begin{aligned}
& A_{\delta, i}=\lim _{(|u|+|v|) \rightarrow \delta} \frac{f_{i}(u, v)}{(|u|+|v|)^{p-1}},\left(\delta=0^{+} \text {or }+\infty\right), \\
& E_{i}=\int_{0}^{1} G_{i}(s, s) d s, \\
& F_{i}=\frac{a_{i}^{q-1}}{(\Gamma(\alpha))^{q-1}} \int_{0}^{1} G_{i}(s, s)\left(\int_{s}^{1}(\tau-s)^{\alpha-1} d \tau\right)^{q-1} d s, \text { where } a_{i}=\max _{t \in[0.1]} a_{i}(t)
\end{aligned}
$$


By simple calculation, we get

$$
\begin{aligned}
& E_{i}=\frac{\eta_{i}}{\left(\eta_{i}-1\right) \Gamma\left(\beta_{i}+1\right)} \\
& F_{i}=\frac{\eta_{i} a_{i}^{q-1}}{\left(\eta_{i}-1\right)(\Gamma(\alpha+1))^{q-1} \Gamma\left(\beta_{i}\right)\left[\alpha(q-1)\left(\beta_{i}-1\right)+1\right]}, i=1,2
\end{aligned}
$$

Positive solutions for p-Laplacian systems

We make the following assumption:

$(\mathrm{H})$ : There exist two nonnegative functions $c_{1}, c_{2} \in L^{1}[0,1]$ and two constants $b_{1}, b_{2}>0$ such that

$$
g_{1}(t, u, v) \leq b_{1} c_{1}(t)(u+v), \quad g_{2}(t, u, v) \leq b_{2} c_{2}(t)(u+v),
$$

for $(u, v) \in \mathbb{R}^{+} \times \mathbb{R}^{+}$, with $\left\|c_{i}\right\|_{L^{1}} \leq \frac{\eta_{i}-1}{2 b_{i}}, i=1,2$.

Lemma 4.1. The system $(S)$ has a positive solution $(u, v)$ if and only if $(u, v)$ is a positive solution for the following system of integral equations:

$$
\left\{\begin{array}{l}
u(t)=\int_{0}^{1} G_{1}(t, s)\left(\frac{1}{\Gamma(\alpha)} \int_{s}^{1}(\tau-s)^{\alpha-1} a_{1}(\tau) f_{1}(u(\tau), v(\tau)) d \tau\right)^{q-1} d s \\
+\frac{1}{\eta_{1}-1} \int_{0}^{1} g_{1}(s, u(s), v(s)) d s \\
v(t)=\int_{0}^{1} G_{2}(t, s)\left(\frac{1}{\Gamma(\alpha)} \int_{s}^{1}(\tau-s)^{\alpha-1} a_{2}(\tau) f_{2}(u(\tau), v(\tau)) d \tau\right)^{q-1} d s \\
+\frac{1}{\eta_{2}-1} \int_{0}^{1} g_{2}(s, u(s), v(s)) d s .
\end{array}\right.
$$

Proof. Easily obtained by Lemma 3.2, then we omit it.

Define the operator

$$
\begin{array}{r}
T: P \rightarrow C[0,1] \times C[0,1] \\
T(u, v)=\left(T_{1}(u, v), T_{2}(u, v)\right),
\end{array}
$$

where $T_{i}: P \rightarrow C[0,1]$ and

$$
\begin{aligned}
T_{i}(u, v)= & \int_{0}^{1} G_{i}(t, s)\left(\frac{1}{\Gamma(\alpha)} \int_{s}^{1}(\tau-s)^{\alpha-1} a_{i}(\tau) f_{i}(u(\tau), v(\tau)) d \tau\right)^{q-1} d s \\
& +\frac{1}{\eta_{i}-1} \int_{0}^{1} g_{i}(s, u(s), v(s)) d s .
\end{aligned}
$$


AJMS

27,2

\section{2}

Then, by Lemma 4.1, the existence of solutions for problem $(S)$ is translated into the existence of fixed points for $T(u, v)=(u, v)$, thus the fixed point of the operator $T$ coincides with the solution of problem $(S)$.

Lemma 4.2. Let $T: P \rightarrow X$ be the operator defined by (4.3). Then $T$ is completely continuous and $T P \subset P$.

Proof. First, we shall show that $T P \subset P$. We have for each $t \in[0,1]$,

$$
\begin{aligned}
\left|T_{i}(u(t), v(t))\right| \leq & \int_{0}^{1} G_{i}(t, s)\left(\frac{1}{\Gamma(\alpha)} \int_{s}^{1}(\tau-s)^{\alpha-1} a_{i}(\tau) f_{i}(u(\tau), v(\tau)) d \tau\right)^{q-1} d s \\
& +\frac{1}{\eta_{i}-1} \int_{0}^{1} g_{i}(s, u(s), v(s)) d s
\end{aligned}
$$

Taking the supremum over $[0,1]$, we get

$$
\begin{aligned}
\left\|T_{i}(u, v)\right\|_{\infty} \leq & \int_{0}^{1} G_{i}(t, s)\left(\frac{1}{\Gamma(\alpha)} \int_{s}^{1}(\tau-s)^{\alpha-1} a_{i}(\tau) f_{i}(u(\tau), v(\tau)) d \tau\right)^{q-1} d s \\
& +\frac{1}{\eta_{i}-1} \int_{0}^{1} g_{i}(s, u(s), v(s)) d s .
\end{aligned}
$$

On the other side, we have

$$
\begin{aligned}
T_{i}(u(t), v(t)) \geq & \frac{1}{\eta_{i}} \int_{0}^{1} G_{i}(t, s)\left(\frac{1}{\Gamma(\alpha)} \int_{s}^{1}(\tau-s)^{\alpha-1} a_{i}(\tau) f_{i}(u(\tau), v(\tau)) d \tau\right)^{q-1} d s \\
& +\frac{1}{\eta_{i}-1} \int_{0}^{1} g_{i}(s, u(s), v(s)) d s
\end{aligned}
$$

Since $\eta_{i}>1$, then,

$$
T_{i}(u(t), v(t)) \geq \frac{1}{\eta_{i}}\left\|T_{i}(u, v)\right\|_{\infty} .
$$

That is $T P \subset P$.

Second, we shall proof that $T$ is completely continuous that will be done in two steps.

Step 1: By the continuity of the functions $f_{i}$ and $g_{i}$ it yields for $n \geq N$,

$$
\begin{aligned}
& \left|f_{i}\left(u_{n}(\tau), v_{n}(\tau)\right)-f_{i}(u(\tau), v(\tau))\right|<\varepsilon, \\
& \left|g_{i}\left(s, u_{n}(s), v_{n}(\tau)\right)-g_{i}(s, u(s), v(\tau))\right|<\varepsilon .
\end{aligned}
$$


(1) If $1<q \leq 2$, then from Lemma 2.1

$$
\begin{aligned}
& \left|\left(\int_{s}^{1}(\tau-s)^{\alpha-1} a_{i}(\tau) f_{i}(u(\tau), v(\tau)) d \tau\right)^{q-1}-\left(\int_{s}^{1}(\tau-s)^{\alpha-1} a_{i}(\tau) f_{i}(u(\tau), v(\tau)) d \tau\right)^{q-1}\right| \\
& \quad \leq\left(\int_{s}^{1}(\tau-s)^{\alpha-1} a_{i}(\tau)\left|f_{i}\left(u_{n}(\tau), v(\tau)\right)-f_{i}(u(\tau), v(\tau))\right| d \tau\right)^{q-1} \\
& \quad<\left[\frac{\varepsilon}{\alpha} a_{i}\right]^{q-1} .
\end{aligned}
$$

Then,

$$
\begin{aligned}
\left|T_{i}\left(u_{n}, v_{n}\right)-T_{i}(u, v)\right| & <\frac{a_{i}^{q-1} \varepsilon^{q-1}}{(\Gamma(\alpha+1))^{q-1}} \int_{0}^{1} G_{i}(s, s) d s+\frac{\varepsilon}{\eta_{i}-1} \\
& =\frac{a_{i}^{q-1} \varepsilon^{q-1}}{(\Gamma(\alpha+1))^{q-1}} E_{i}+\frac{1}{\eta_{i}-1} \varepsilon .
\end{aligned}
$$

Hence

$$
\left\|T_{i}\left(u_{n}, v_{n}\right)-T_{i}(u, v)\right\|_{\infty} \leq\left(\frac{a_{i}^{q-1} E_{i}}{(\Gamma(\alpha+1))^{q-1}}+\frac{1}{\eta_{i}-1}\right) \varepsilon^{q-1}
$$

(2) If $q>2$, then from Lemma 2.1, we have

$$
\begin{aligned}
& \mid\left(\frac{1}{\Gamma(\alpha)} \int_{s}^{1}(\tau-s)^{\alpha-1} a_{i}(\tau) f_{i}\left(u_{n}(\tau), v(\tau)\right) d \tau\right)^{q-1} \\
& \quad-\left(\frac{1}{\Gamma(\alpha)} \int_{s}^{1}(\tau-s)^{\alpha-1} a_{i}(\tau) f_{i}(u(\tau), v(\tau)) d \tau\right)^{q-1} \mid \\
& \quad \leq \frac{(q-1)(c)^{q-2}}{\Gamma(\alpha)} \int_{s}^{1}(\tau-s)^{\alpha-1} a_{i}(\tau)\left|f_{i}\left(u_{n}(\tau), v(\tau)\right)-f_{i}(u(\tau), v(\tau))\right| d \tau \\
& \quad<\frac{(q-1) c^{q-2} a_{i}}{\Gamma(\alpha+1)} \varepsilon .
\end{aligned}
$$

So,

$$
\left|T_{i}\left(u_{n}, v_{n}\right)-T_{i}(u, v)\right|<\left(\frac{(q-1) c^{q-2} a_{i}}{\Gamma(\alpha+1)} \int_{0}^{1} G_{i}(s, s) d s+\frac{1}{\eta_{i}-1}\right) \varepsilon .
$$


AJMS

27,2

Hence

$$
\left\|T_{i}\left(u_{n}, v_{n}\right)-T_{i}(u, v)\right\|_{\infty}<\left(\frac{(q-1) c^{q-2} a_{i}}{\Gamma(\alpha+1)} E_{i}+\frac{1}{\eta_{i}-1}\right) \varepsilon .
$$

From (4.5)-(4.6) it follows that $\left\|T\left(u_{n}, v_{n}\right)-T(u, v)\right\| \rightarrow 0$ as $n \rightarrow \infty$, thus $T$ is continuous.

Step 2: The operator $T$ is uniformly bounded on $P$. Let $\Omega$ be an open bounded set in $P$. Set

$$
\begin{aligned}
& L_{i}=\max f_{i}(u(t), v(t))<\infty, \quad l_{i}=\max g_{i}(t, u(t), v(t)) . \\
& (u, v) \in \bar{\Omega} \\
& (t, u, v) \in[0,1] \times \bar{\Omega}
\end{aligned}
$$

Then for $(t, u, v) \in[0,1] \times \Omega$, we have

$$
\begin{aligned}
& \left|T_{i}(u(t), v(t))\right| \leq \int_{0}^{1} G_{i}(t, s)\left(\frac{1}{\Gamma(\alpha)} \int_{s}^{1}(\tau-s)^{\alpha-1} a_{i}(\tau) f_{i}(u(\tau), v(\tau)) d \tau\right)^{q-1} d s \\
& \quad+\frac{1}{\eta_{i}-1} \int_{0}^{1} g_{i}(s, u(s), v(s)) d s \\
& \quad \leq\left[\frac{L_{i} a_{i}}{(\Gamma(\alpha+1))}\right]^{q-1} \int_{0}^{1} G_{i}(s, s) d s+\frac{l_{i}}{\left(\eta_{i}-1\right)} \\
& \quad=\left[\frac{L_{i} a_{i}}{(\Gamma(\alpha+1))}\right]^{q-1} E_{i}+\frac{l_{i}}{\eta_{i}-1}<\infty
\end{aligned}
$$

thus $T(\Omega)$ is uniformly bounded.

Now we prove that $T(\Omega)$ equicontinuous, Let $(u, v) \in \Omega, 0 \leq t_{1} \leq t_{2} \leq 1$. We have

$$
\begin{aligned}
& \left|T_{i}\left(u\left(t_{1}\right), v\left(t_{1}\right)\right)-T_{i}\left(u\left(t_{2}\right), v\left(t_{2}\right)\right)\right| \\
& \quad \leq \int_{0}^{t_{1}}\left|G_{i}\left(t_{2}, s\right)-G_{i}\left(t_{1}, s\right)\right|\left(\frac{1}{\Gamma(\alpha)} \int_{s}^{1}(\tau-s)^{\alpha-1} a_{i}(\tau) f_{i}(u(\tau), v(\tau)) d \tau\right)^{q-1} d s \\
& \quad+\int_{t_{2}}^{1}\left|G_{i}\left(t_{2}, s\right)-G_{i}\left(t_{1}, s\right)\right|\left(\frac{1}{\Gamma(\alpha)} \int_{s}^{1}(\tau-s)^{\alpha-1} a_{i}(\tau) f_{i}(u(\tau), v(\tau)) d \tau\right)^{q-1} d s \\
& \quad+\int_{t_{1}}^{t_{2}}\left|G_{i}\left(t_{2}, s\right)-G_{i}\left(t_{1}, s\right)\right|\left(\frac{1}{\Gamma(\alpha)} \int_{s}^{1}(\tau-s)^{\alpha-1} a_{i}(\tau) f_{i}(u(\tau), v(\tau)) d \tau\right)^{q-1} d s \\
& \quad \leq\left[\frac{L_{i} a_{i}}{(\Gamma(\alpha+1))}\right]^{q-1} \frac{\left|t_{2}-t_{1}\right|^{\beta_{i}}}{\Gamma\left(\beta_{i}+1\right)} .
\end{aligned}
$$

Consequently, $\left|T_{i}\left(u\left(t_{1}\right), v\left(t_{1}\right)\right)-T_{i}\left(u\left(t_{2}\right), v\left(t_{2}\right)\right)\right| \rightarrow 0$, when $t_{2} \rightarrow t_{1}$. Hence $T(\Omega)$ is equicontinuous. Finally, by Arzela-Ascoli's theorem, it follows that $T$ is completely continuous mapping on $\Omega$. 
Theorem 4.1. Assume that the condition $(H)$ is satisfied, then the system $(S)$ has at least one nontrivial positive solution $(u, v)$ in the cone $P$, in the case $A_{0, i}=0$ and $A_{\infty, i}=\infty, i=1,2$.

Proof. From $A_{0, i}=0, i=1,2$, we deduce that for

$$
0<\varepsilon \leq \min _{i=1,2}\left\{\left[\left(1-\frac{b_{i}}{\eta_{i}-1}\left\|c_{i}\right\|_{L^{1}}\right) \frac{1}{F_{i}}\right]^{\frac{1}{q-1}}\right\}
$$

Positive solutions for p-Laplacian systems

there exist $\rho_{1}>0$, such that if $0<u+v \leq \rho_{1}$, then

$$
f_{i}(u, v) \leq \varepsilon(|u|+|v|)^{p-1}
$$

Let $\Omega_{1}=\left\{(u, v) \in X,\|(u, v)\|<\rho_{1}\right\}$. Assume that $(u, v) \in P \cap \partial \Omega_{1}$, then

$$
\begin{aligned}
& T_{i}(u(t), v(t)) \leq \int_{0}^{1} G_{i}(s, s)\left(\frac{1}{\Gamma(\alpha)} \int_{s}^{1}(\tau-s)^{\alpha-1} a_{i}(\tau) \varepsilon(|u|+|v|)^{p-1} d \tau\right)^{q-1} d s \\
& \quad+\frac{1}{\eta_{i}-1} \int_{0}^{1} b_{i} c_{i}(s)(|u|+|v|) d s . \\
& \leq\left(\frac{\varepsilon}{\Gamma(\alpha)}\right)^{q-1} \int_{0}^{1} G_{i}(s, s) \\
& \quad \times\left(\int_{s}^{1}(\tau-s)^{\alpha-1} a_{i}(\tau)\left(\|u\|_{\infty}+\|v\|_{\infty}\right)^{p-1} d \tau\right)^{q-1} d s \\
& \quad+\frac{b_{i}}{\eta_{i}-1} \int_{0}^{1} c_{i}(s)\left(\|u\|_{\infty}+\|v\|_{\infty}\right) d s . \\
& \quad=\|(u, v)\|\left(\varepsilon^{q-1} F_{i}+\frac{b_{i}}{\eta_{i}-1}\left\|c_{i}\right\|_{L^{1}}\right) \\
& \leq\|(u, v)\| .
\end{aligned}
$$

Hence

$$
\|T(u, v)\| \leq\|(u, v)\|, \text { for }(u, v) \in \partial \Omega_{1} \cap P
$$

Since $A_{\infty, i}=\infty, i=1,2$, so for

$$
\mu \geq \max _{i=1,2}\left\{\left(\frac{\eta^{2} \Gamma(\alpha)}{\xi_{i}}\right)^{\frac{1}{q-1}}\right\}, \xi_{i}=\int_{0}^{1} G_{i}(s, s)\left(\int_{s}^{1}(\tau-s)^{\alpha-1} a_{i}(\tau) d \tau\right)^{q-1} d s,
$$


there exists $\rho>0$, such that if $u+v \geq \rho$, then

$$
f_{i}(u, v) \geq \mu(|u|+|v|)^{p-1} .
$$

Let $\rho_{2}=\max \left(\frac{3}{2} \rho_{1}, \eta \rho\right), \eta=\max \left(\eta_{1}, \eta_{2}\right)$, and set $\Omega_{2}=\left\{(u, v) \in X,\|(u, v)\|<\rho_{2}\right\}$, it is easy to see that $\Omega_{1} \subset \Omega_{2}$. Assume that $(u, v) \in P \cap \partial \Omega_{2}$, then

$$
\begin{aligned}
& T_{i}(u(t), v(t)) \geq \frac{1}{\eta_{i}} \int_{0}^{1} G_{i}(s, s)\left(\frac{1}{\Gamma(\alpha)} \int_{s}^{1}(\tau-s)^{\alpha-1} a_{i}(\tau) \mu(|u|+|v|)^{p-1} d \tau\right)^{q-1} d s \\
& \geq \frac{1}{\eta_{i}}\left(\frac{\mu}{\Gamma(\alpha)}\right)^{q-1} \int_{0}^{1} G_{i}(s, s) \\
& \quad \times\left(\int_{s}^{1}(\tau-s)^{\alpha-1} a_{i}(\tau)\left(\frac{1}{\eta_{1}}\|u\|_{\infty}+\frac{1}{\eta_{2}}\|v\|_{\infty}\right)^{p-1} d \tau\right)^{q-1} d s \\
& \geq \frac{1}{\eta^{2}}\left(\frac{\mu}{\Gamma(\alpha)}\right)^{q-1} \xi_{i}\|(u, v)\| \geq\|(u, v)\|,
\end{aligned}
$$

thus

$$
\|T(u, v)\| \geq\|(u, v)\|,(u, v) \in \partial \Omega_{2} \cap P .
$$

By Guo-Krasnosel'skii fixed-point theorem, we conclude that $T$ has a fixed point $(u, v) \in P \cap\left(\bar{\Omega}_{2} \backslash \Omega_{1}\right)$. This means that the system $(S)$ has at least one positive solution $(u, v)$.

Example 4.1. Consider the system $(S)$, with

$$
\begin{aligned}
& f_{1}(u, v)=(u+v)^{3}, f_{2}(u, v)=e^{(u+v)^{2}}-1 \\
& a_{1}(t)=e^{t}, a_{2}(t)=1 \\
& g_{1}(t, u, v)=\frac{(1-t)(u+v)^{2}}{3 u+4 v}, g_{2}(t, u, v)=\frac{t}{9} u
\end{aligned}
$$

where $\alpha=\frac{1}{2}, \beta_{1}=\beta_{2}=\frac{4}{3}, p=2, \eta_{1}=\frac{3}{2}, \eta_{2}=\frac{5}{4}$. We check easily that $A_{0, i}=0, A_{\infty, i}=\infty$, $i=1,2$. Clearly,

$$
g_{1}(t, u, v) \leq \frac{1-t}{3}(u+v), g_{2}(t, u, v) \leq \frac{t}{5}(u+v)
$$

So, the assumption $(H)$ hold. Thus the system $(S)$ has at least one positive solution by Theorem 4.1.

\section{References}

[1] Guo DJ, Lakshmikantham V. Nonlinear problems in abstract cones in: notes and reports in mathematics in science and engineering, Boston, Mass: Academic Press; 1988; 5.

[2] Kilbas AA, Srivastava HM, Trujillo JJ. Theory and applications of fractional differential equations. North-Holland mathematics studies, Amsterdam: Elsevier Science B.V., 2006; 204. 
[3] Podlubny I. Fractional differential equations, San Diego: Academic Press; 1999.

[4] Guezane Lakoud A, Khaldi R, K1lıçman A. Existence of solutions for a mixed fractional boundary value problem. Adv Differ Equ. 2017; 2017, 164.

[5] Khaldi R, Guezane-Lakoud A. Higher order fractional boundary value problems for mixed type derivatives. J Nonlinear Funct Anal. 2017; 2017, 30.

[6] Bashir A, Nieto JJ. Existence of solutions for anti-periodic boundary value problems involving fractional differential equations via Leray-Schauder degree theory. Topol Methods Nonlinear Anal. 2010; 35: 295-304.

[7] Cabada A, Wang G. Positive solutions of nonlinear fractional differential equations with integral boundary value conditions. J Math Anal Appl. 2012; 389: 403-11.

[8] Ding Y, Xu J and Fu Z. Positive solutions for a system of fractional integral boundary value problems of Riemann-Liouville type involving Semipositone nonlinearities. Mathematics. 2019; 7: 970. 10.3390.

[9] Guezane-Lakoud A, Khaldi R. Positive solutions for multiorder nonlinear fractional systems. Int J Anal Appl. 2017; 15: 18-22.

[10] Guezane-Lakoud A, Ramdane S. Existence of solutions for a system of mixed fractional differential equations. J Taibah Univ Sci. 2018; 12. doi: 10.1080/16583655.2018.1477414.

[11] Khaldi R, Guezane-Lakoud A. Solvability of a boundary value problem with a Nagumo condition. J Taibah Univ Sci. 2018. doi: 10.1080/16583655.2018.1489025.

[12] Li Y, Wei Z. Positive solutions for a coupled system of mixed higher-order nonlinear singular fractional differential equations. F Point Theory. 2014; 15(1): 167-78.

[13] Yang W. Positive solutions for a coupled system of nonlinear fractional differential equations with integral boundary conditions. Comput Math Appl. 2012; 63: 288-97.

[14] Guezane-Lakoud A, Ashyralyev A. Fixed point theorem applied to a fractional boundary value problem. Pure App Math Letters. 2014; 2: 1-6.

[15] Ahmad B, Nieto JJ. Existence results for a coupled system of nonlinear fractional differential equations with three-point boundary conditions. Comput Math Appl. 2009; 58(9): 1838-43.

[16] Bai C, Fang J. The existence of a positive solution for a singular coupled system of nonlinear fractional differential equations. Appl Math Comput. 2004; 150(2): 611-21.

[17] Liu Y. A new method for converting boundary value problems for impulsive fractional differential equations to integral equations and its applications. Adv Nonlinear Anal. 2019; 8(1): $386-54$.

[18] Agarwal RP, Liu HS and O'Regan D. Existence theorems for the one-dimensional singular p-Laplacian equation with sign changing nonlinearities. Appl Math Comput. 2003; 143: 15-38.

[19] Chen T, Liu WB. An anti-periodic boundary value problem for the fractional differential equation with a p-Laplacian operator. Appl Math Lett. 2012; 25: 1671-75.

[20] Chen T, Liu WB and Hu ZG. A boundary value problem for fractional differential equation with p-Laplacian operator at resonance. Non-linear Anal. 2012; 75: 3210-17.

[21] Han Z, Lu H, Sun S, Yang D. Positive solutions to boundary -value problems of P-Laplacian fractional differential equations with a parameter in boundary. Elec Jou Diff Equa. 2012; 2012(213): 1-14.

[22] Mahmudo NI, Unul S. Existence of solutions of fractional boundary value problems with pLaplacian operator. Bound. Value Probl. 2015; 99. doi 10.1186/s13661-015-0358-9.

[23] Tian Y, Bai Z, Sun S. Positive solutions for a boundary value problem of fractional differential equation with p-Laplacian operator. Tian et al. Adv Differ Equ. 2019; 349.

[24] Wang J, Xiang H. Upper and lower solutions method for a class of singular fractional boundaryvalue problems with p-Laplacian operator. Abs Appl Anal. 2010; 2010, 971824; 1-12.

\section{Positive solutions for p-Laplacian systems}


AJMS

27,2

\section{8}

[25] Wang J, Xiang H, Liu Z. Existence of concave positive solutions for boundary-value problem of nonlinear fractional differential equation with p-Laplacian operator. Int J Math Math Sci. 2010: 2010, 495138: 1-17.

[26] Chai GQ. Positive solutions for boundary value problem of fractional differential equation with p-Laplacian operator, Bound Value Probl. 2012; 2012: 18. doi: 10.1186/1687-2770-2012-18.

[27] Krasnosel'skii MA. Positive solutions of operator equations: Groningen; 1964.

\section{Further reading}

[28] Agrawal OP. Formulation of Euler-Lagrange equations for fractional variational problems. J Math Anal Appl. 2002; 272: 368-79.

[29] Guezane-Lakoud A, Khaldi R, Torres DFM. On a fractional oscillator equation with natural boundary conditions. Prog Frac Diff Appl. 2017; 3: 191-7.

[30] Podlubny I. Geometric and physical interpretation of fractional integration and fractional differentiation. Fract Calc Appl, Anal. 2002; 5: 367-86.

[31] Samko SG, Kilbas AA, Marichev OI. Fractional integrals and derivatives: theory and applications. Yverdon: Gordon and Breach; 1993.

[32] Xie S. Positive solutions for a system of higher-order singular nonlinear fractional differential equations with nonlocal boundary conditions. E. J. Qualitative Theory of Diff Equa. 2015; (18): $1-17$.

\section{Corresponding author}

Samira Ramdane can be contacted at: s_ramdane2016@yahoo.com

For instructions on how to order reprints of this article, please visit our website:

www.emeraldgrouppublishing.com/licensing/reprints.htm

Or contact us for further details: permissions@emeraldinsight.com 